\title{
Attendants' Satisfaction with Child Immunization Service in Primary Health Centers in New Cairo District-Egypt
}

\author{
Sahar A Dewedar*, Nahla F Abou El Ezz*, Iman M A Bakr*, Elham H. \\ Ahmed** \\ *Community, Environmental and Occupational Medicine \\ Faculty of Medicine - Ain Shams University \\ **Family Health Centers of New Cairo District, MOHP, Cairo, Egypt. \\ Received: July 2015, accepted: October 2015
}

\begin{abstract}
Introduction: Childhood immunization is considered to be among the most effective preventive services, and is therefore critical to monitor and evaluate. Objectives: The study was conducted to determine attendants' satisfaction with child immunization services in four primary health care centers of New Cairo district; also skills check list to evaluate the immunization service was done. Methodology: A cross section study using an interview questionnaire was conducted on 550 attendants from four primary health care centers in new Cairo district. Systematic random sample of attendants every $7^{\text {th }}$ attendant was interviewed after finishing his service to ascertain his levels of satisfaction with immunization service. Results: the highest satisfaction was reported in the resource availability and to immunization as a service; it was $94.9 \%$ and $96 \%$ respectively). The least satisfaction was from waiting time and vaccine session, it was $62.3 \%$ and $51.4 \%$ respectively. Overall satisfaction in the present study was $74.4 \%$. Highest satisfaction was reported to immunization as a service it was $96 \%$ and least satisfaction was recorded from the vaccine session $51.4 \%$.
\end{abstract}

Keywords: Primary health care, Childhood immunization, client satisfaction.

*corresponding author, Elham Hemdan Ahmed Email: ahmed_zedan2007@yahoo.com

\section{Introduction}

Primary health care is a part of health care very important and serve a big layer of the society, especially those of low socioeconomics, also there is a services not present in the medical part of the health care like immunization.

Primary health care "PHC" has been defined as essential care based on practical scientifically sound and socially acceptable methods and technology made universally accessible to individuals and families in the community through their full participation and at cost that the community and the country can afford (1).

Primary health care include basic child health services such as vaccination service.

The World Health Organization (WHO) identified the need for public health intervention and in 1974 initiated the expanded program of Immunization (EPI), which aims to immunize, and thus protect, mothers against Tetanus and their children against the following six deadly diseases: Childhood 
tuberculosis, Poliomyelitis, Diphtheria, Pertussis, Measles and Tetanus (2).

Childhood immunization considered to be among the most effective preventive services, and is therefore critical to monitor and evaluate ${ }^{(3)}$

Timely immunization depends on both appropriate access to health care and the administration of eligible doses at each visit (4).

Immunization remains one of the most important public health interventions and a cost effective strategy to reduce both the morbidity and mortality associated with infectious diseases. Over two million deaths are delayed through immunization each year worldwide $^{(\mathbf{6})}$. Despite this, vaccine preventable diseases remain the most common cause of childhood mortality with an estimated three million deaths each year ${ }^{(6)}$

Attendants' satisfaction with health care is important for consistent relationships with care providers, identifying source of dissatisfaction, improved compliance, continuity of care, and ultimately better health outcomes $^{(7)}$

Attendants' satisfaction is an important measure of service quality in health care organizations. determining the level of patient satisfaction has been found to be the most useful tool for getting patient's view on how to provide care ${ }^{(8)}$.

Many studies were done to measure satisfaction in Egypt in 2003 , a study was done to compare satisfaction in a primary health care unit in lower Egypt (Belbies- Elsharquia) \& in upper Egypt( Abou Kourkas -Elmenia), and the degree of satisfaction among them was $97 \% \& 98 \%$ respectively ${ }^{(9)}$.

\section{Study hypothesis:}

Decreased attendants' satisfaction of immunization health care services among attendants of primary health centers in New Cairo district.

\section{Study question.}

What is the level of attendants' satisfaction with child immunization service in primary health centers in New Cairo district.

\section{Objectives:}

1-Ultimate objective is to Increase attendants' satisfaction with primary health services.

2-Spcifice objectives to Measure attendant satisfaction with child immunization service in primary health care centers of New Cairo district.

*To Evaluate child immunization service in primary health care centers of New Cairo district.

\section{Methodology}

\section{Type of the study :}

A cross-sectional study was done that included a sample of outpatient parents attending the vaccination session of their children in the 4 primary health care units in New Cairo over three months duration .

\section{Study population:}

A sample of parents coming to the health care units will be chosen during the vaccination session of their children.

\section{Sample size:-}

A sample size was calculated using EPI-info 2002 was found to be 500 of attendants was estimated at $95 \%$ C.I. 
taking a level of satisfaction among attendants of $64.2 \%{ }^{(25)}$ putting in consideration $\alpha$ error $=(0.05) 10 \%$ was added to compensate for refusal to give a final sample 550 .

\section{Sampling:}

The sample was collected over three days per week for three months

A systematic random sample of attendants was followed. Every $7^{\text {th }}$ client was interviewed after receiving the service to ascertain the levels of satisfaction with the health care service. Also evaluation of the immunization service was conducted in the PHCs of the study,

Total number of subjects of 550 were interviewed. The sample was divided according to percent of attendance in the different centers, from the $1^{\text {st }}$ settlement center $221(40.2 \%)$ from the $3^{\text {rd }}$ settlement center $110(20.0 \%)$ from the $5^{\text {th }}$ settlement A center $129(23.5 \%)$ and the $5^{\text {th }}$ settlement $B$ center 90 $(16.4 \%)$ attendants.

\section{Study tools:-}

\section{For the first objective}

An interview questionnaire was used, the questionnaire included two parts:

- Questions about sociodemographic characters such as sex, age, nationality, marital status, job, education level and the income.

- questions about 7 domains of satisfaction were collected

1- Waiting time, 2-Waiting place, 3-Accessibility to the service, 4Vaccination session, 5-Resource availability, 6-Nurse performance and 7-Satisfaction to immunization as a service.

A scale was used in each question as ( very good - good -bad - very bad - don't know. They were coded as 4,3,2, 1 , and 0 respectively) as for the (yes no - don't know) questions they were coded as (2,1 and 0 respectively) .

A total score was calculated to determine the overall satisfaction for the seven domains

\section{For the second objective}

A checklist was used to assess the following points.

1- Communication skills and providing vaccination knowledge to attendant.

2- Medical protocols applied inside the vaccination room,

3- Vaccine handling,

4- Protocols for administering immunization,

5- Records filling procedures.

A scale was used for each statement in the check list (done -not properly donenot done. they were coded as 2,1 , and 0 respectively)

Check list for evaluation of the cold chain and health care worker performance $^{(16)}$

1- Assessment of cold chain and proper storage.

2- Assessment of performance of health workers in vaccination session.

A scale was used (No - Yes coded in 0 and 1 respectively).

\section{Data analysis:}

Data was collected; coded and analyzed using (SPSS) program and appropriate statistical tests were used.

- Quantitative data are presented as mean \pm standard deviation.

- Qualitative data are presented as number and percentage.

$\begin{array}{llll}\text { Vol. } 34 & \text { No. } 3 & \text { July } & 2016\end{array}$


- Chi-squared test is used to compare such data between two or more groups.

\section{Ethical consideration:}

1- Administrative approval for conducting this research was obtained then the permission from the authorities of the health unit included in the study was collected.

2- Assuring confidentiality of information, data collected was anonymous.

3- Subjects were informed about the study objectives and procedures.

4- Verbal consents were obtained from all participant

\section{Results:-}

\section{Attendant satisfaction in the four PHCs}

Regarding satisfaction with waiting time the highest satisfaction was found among attendants of the st settlement center $(72.7 \%)$ while the least satisfaction was recorded among attendants of the $5^{\text {th }}$ A Center $(55.0 \%)$ however the difference was not statistically significant as shown in (table 1).

For the waiting place the least satisfaction was reported in the $5^{\text {th }} \mathrm{A}$ settlement. Center as regard the waiting place ventilation $(60.6 \%)$, while waiting place space $(68.2 \%)$, waiting place lighting was $(42.6 \%)$ and as regard absence of chairs was $(81.4 \%)$ the difference between this center and others 3 centers was statistically significant $(\mathrm{P}$ value $<0.001)$ as shown in(table 1).

As regard office ventilation the least satisfaction was in the $5^{\text {th }}$ settlement Center B (64.4\%) and the difference between this center and others was statistically significant ( $\mathrm{P}$ value $<0.001)$ as shown in(table 1).

Regarding accessibility the least satisfaction among attendants was in the $5^{\text {th }} \quad$ sett. Center A as regard immunization office location $(62 \%)$. to illustrating posters $(31.8 \%)$ and availability of transportation (79.8\%). the difference in attendant satisfaction between this center and other 3 was statistically significant $(\mathrm{P}$ value 0.009 and <0.001 respectively) as showed in(table 1).

Also the $5^{\text {th }}$ sett. Center A showed the least satisfaction among attendants as regard the long distance to reach the center $(55.8 \%)$ and having information about the vaccination days $(75.2 \%)$, however the difference was not statistically significant when comparing with other 3 PHCs as shown in(table 1).

Regarding nurse performance there was no statistical difference in the satisfaction with assessment of nurse performance in the 4 centers except for side effects explained as the least satisfaction was in the $5^{\text {th }}$ center $A$ (88.4\%), however there was statistically significant difference between this center and other 3 centers. Also as regard hand washing of the nurse the least satisfaction was in the $5^{\text {th }} \mathrm{A}$ and $5^{\text {th }}$ centers B (20\%). however there was statistically significant difference between these 2centers and other 2 centers $(\mathrm{P}$ value <0.001) as shown in(table 1).

For resource availability the $5^{\text {th }}$ sett. Center A had the least satisfaction as a significantly higher percentage $(19.4 \%)$ of attendants in the $5^{\text {th }}$ center A brought external vaccination supplies compared to the other 3 centers and the difference was statistically significant $(\mathrm{P}$ value $<0.001)$ as shown in(table 1$)$. 
As regard education the non educated were more satisfied than the educated as shown in (figure 1).

Finally overall satisfaction from the service was $(74.4 \%)$ as shown in (table1) .

\section{Regarding evaluation of immunization service}

The $1^{\text {st }}$ settlement Center had the highest percentage in all skills of evaluating check list, while the 5th sett. Center B had the lowest percentage in all skills and there was highly significant difference in performing these skills ( $\mathrm{P}$ value $=0.00)$ as shown in(table 2).

The four centers had similar performance in vaccination preservation $(100 \%)$ while as regards the performance of health care workers the $1^{\text {st }}$ and $3^{\text {rd }}$ sett. Centers had the highest similar percentage compared to other 2 centers with significant difference $(\mathrm{P}$ value $<0.00)$ as shown in (table 3).

\section{Discussion}

\section{Attendant satisfaction in the four PHCs}

Attendants' satisfaction is now an important indicator of the service delivered in primary health care. In this study attendant satisfaction of child immunization service in four PHCs of new Cairo district was assessed.

All aspects of immunization attendants' satisfaction were compared in the four Primary health care centers.

Here are the deferent domains of satisfaction:

In the present study satisfaction with waiting time was (62.32) this was similar to what was reported in an Egyptian study in Ismailia Governorate primary health care center about satisfaction from waiting time which was $(61.5 \%)^{(10)}$ this due to the same cultural behaviors of the people.

In a Nigerian study satisfaction from waiting time was $(49 \%)^{(12)]}$ and in a study done in Guatemala satisfaction to waiting time was $(59 \%)^{(\mathbf{1 8})}$ this was less than that found in the present study this difference may be due to low number of the attendants of the present study as the study was done in a new area that was not crowded or different believes and culture.

In a study done in Qassim Province, Saudi Arabia satisfaction to waiting time was $(72 \%)$ which was better than what reported in the present study(12).

In the present study satisfaction to waiting place was $(77.07 \%)$ it was better than what was reported in an Egyptian study in Ismailia Governorate primary health care center as the satisfaction was $(62.9 \%)^{(10)}$, a Nigerian study reported less satisfaction from waiting place $(49 \%)^{(\mathbf{1 1})}$ this may be due to cultural difference between the two populations in the two studies.

In The present study the highest satisfaction from cleanliness was among attendants of the fifth settlement center B $(91.11 \%)$ and lowest was in the first settlement $(88.18 \%)$ it was much better than what was reported in a Nigerian study as satisfaction was $(58.2 \%)^{(12)}$ this may be due to regular inspection from the infection control team which were regular and effective in the last years but ${ }^{(11)}$ reported that satisfaction to cleanliness was between $(95.5 \%$ and $98.7 \%)$ which was better than what reported in the present study.

Regarding to accessibility satisfaction in this study was (73.39\%) it was similar to what reported In a study done in Qassim Province, Saudi Arabia

No. 3

July 2016 
satisfaction to accessibility was $(72.7 \%)$ (12).

In an Egyptian study done in two districts in Upper and Lower Egypt as satisfaction to accessibility was between $(83 \% \text { and } 91 \%)^{(9)}$ also an Indian study as satisfaction from accessibility was between $(93.94 \%$ 97.8\%) $\left.{ }^{(13}\right)$ this was better than what reported in the present study this difference may be due to availability and good transportation to the centers.

Satisfaction to nurse performance was $(65.3 \%)$ it was higher than what found in a Nigerian study as satisfaction was $(62.6 \%)^{(11)}$ may be due to cultural difference OR regular training of nurses but less than what reported in an Egyptian study done in two districts in upper and lower Egypt as satisfaction to health car providers was $(97.7 \%){ }^{(9)}$.

In an Indian study satisfaction from staff workers was between $(96,6 \%$ 99.6) ${ }^{(13)}$ it was better than what was mentioned in the present study so training programs are recommended to improve practice of much nurses in our PHCs.

According to resource availability highest satisfaction was among the attendants of third settlement $(99.55 \%)$ center and lowest was in the Fifth settlement Center A (90.3\%). it was similar to what was found in an Indian study as satisfaction to resource availability was between (89.5\%$91.6 \%)^{(\mathbf{1 3})}$.

In the present study satisfaction from availability of vaccines was high in all the studied centers $(94.86 \%)$ it was better than what found in an Indian study as satisfaction was between $(89.5 \%-91.6 \%)^{(13)}$ this may be due to care of Egyptian ministry of health to supply the vaccines necessary for the expanded program of immunization.
In the present study satisfaction from the immunization service ranged from (94.4 to $97.27 \%$ ) this was similar to what was reported in an Egyptian study in Ismailia Governorate primary health care center the satisfaction from immunization service was $(95.2 \%){ }^{(\mathbf{1 0})}$. In a Nigerian study satisfaction from immunization service was ranging between $(87 \%-95 \%)^{(\mathbf{1 4})}$ which was less than what was found in this study this difference in satisfaction from the service may be due to good planning of the service in the different health care centers.

Finally overall satisfaction from the service was $(74.4 \%)$ this finding was less than what was reported in the Indian study as the overall satisfaction was between $(91.9 \%-95.9 \%)^{(\mathbf{1 3})}$ high satisfaction from waiting time, staff workers and easy accessibility in the Indian study may have created this difference

\section{Evaluation of immunization service:-}

Regarding communication skills and providing vaccination knowledge to attendants (parent education):-

- For welcoming attendants receiving the service this skill was done by $(62.5 \%)$ in the four centers of our study it is similar to what was reported in an Indian study (65\%) in 26 health facility in Andhra Pradesh area $^{(17)}$

- For explaining vaccines and its routs the percentage of our study was $(62.5 \%)$ it was better than what was presented in the Indian study it was $(50 \%)$. May be due to regular nurse training by the ministry of health.

- For asking about contraindications for immunization in the present

No. 3

July

2016 
study it was (75\%) it is better than what was mentioned in the Indian study $(42 \%)$

\section{Regarding vaccine handling by the nurse during immunization time:-}

- For Checking vial expiration date and double checks vial label this skill done by $(100 \%)$ in the four centers of present study more better than what presented in the Indian study $(81 \%)$. This is because this talent is stressed upon from the ministry of health.

- For Maintains aseptic technique during the vaccination time, in this study it was found in $(87.5 \%)$ this was similar to what was presented in the Indian study $(85 \%)$.

\section{Regarding vaccine administration:-}

- For washing hands before injection (using gloves if in the policy) this was done by $(50 \%)$ in the present study it is better than what was present in the Indian study (31\%). This may be because of regular training of the PHC nurse

- For Injecting vaccine using correct route for the vaccine (ID, SC, IM) it was done by $(100 \%)$ in the present study, better than what was presented in the Indian study (88\%) This may be because of regular training of the PHC nurse

- For positioning and restraining the child with parent's help; locates anatomic landmarks specific for IM or SC this skill was done by $(62.5 \%)$ in the present study it was less than what was reported in the Indian study $(81 \%)$

\section{Regarding records procedures:}

- For Full documentation of each immunization in the patient's chart it was done by (100\%) in the present study better than what was presented in the Indian study (92\%).

Regarding medical protocols inside vaccination room:

- The highest percentage for identifying and performing these protocols was in the $1^{\text {st }}$ settlement Center $(75 \%)$, the least was in the $5^{\text {th }} \mathrm{B}$ settlement Center (50\%).

\section{Two skills were not done:}

1- Preps the site with an alcohol wipe using a circular motion from the center to a 2" to $3 "$ circle. Allows alcohol to dry.

2- Report any needle stick injury to the nurse

Level of vaccine preservation (cold chain and proper storage of vaccines inside it) in the present study was $(100 \%)$

Dewedar (1995) ${ }^{(16)}$ reported in an Egyptian study done in eleven PHCs in Suez governorate that the level of vaccine preservation was (90.9\%) which was less than the level of present study $(100 \%)$.

Mithun(2013) ${ }^{(15)}$ reported in a crosssectional study conducted in Jamnagar district in India from March 2010 to March 2012 in 14 health facility that, functional thermometer inside the fridge and twice daily recording of temperature in a log book was present in $(100 \%)$ this was similar to what was found in this study. As regards vaccine vials placed correctly and arranged inside the fridge it was reported $(85.71 \%)$ this was less than what was found in this study $(100 \%)$.

\section{-Performance of health workers to vaccination process}

*Regarding the written instructions for the system of work inside vaccination

No. 3

July 2016 
room it was present in half of the units in aproper manner.

Dewedar (1995) reported in an Egyptian study done in eleven PHCs in Suez governorate that less than half of the units $(45.5 \%)$ had written instructions for the system of work in a proper manner.

*Regarding registration of vaccinated and non vaccinated children it was done in $50 \%$ of units while in (Dewedar, 1995) (16) it was found that registration of vaccinated and non vaccinated children was done in $100 \%$ of the units.

*Assessment of infant condition before vaccination, it was not done in the four centers of the present study as there was no well baby clinic but (Dewedar, 1995) ${ }^{(\mathbf{1 6})}$ found that it was done in a low level in most centers of the study (64\%).

\section{Recommendations}

1- Regular auditing on cold chain, immunization sessions and satisfaction of parents.

2- Regular Training of the immunization team, training of nurses on communication skills and how to give the immunization, precautions before, after and during also side effects of each vaccine.

3- Improve the registration system of immunization.

4- Another researches recommended to identifies the causes of decreased satisfaction in the $5^{\text {th }}$ sett. center A and decreased evaluation in the $5^{\text {th }}$ sett. Center B.

\section{References}

1)World Health Organization: Declaration of Alma-Ata. Adopted at the International Conference on Primary Health Care, Alma-Ata, USSR, 6-12 September.
2-)Bates AS, Wolinsky FD): Personal, financial, and structural barriers to immunization in socio economically disadvantaged urban children. Pediatrics.1998; 101:591-596.

3)Prislin R, Dyer JA, Blakely $\mathbf{C H}$, Johnson CD: Immunization status and sociodemographic characteristics: the mediating role of beliefs, attitudes, and perceived control. Am J Public Health.1998; 88:1821-1826.

4)Rosenstock IM: Historical origin of the Health Belief Model. Health Educ Monogr.1999 ;2(4):328-35.

\section{5)Luman ET, McCauley MM, Shefer}

A, Chu SY. Maternal characteristics associated with vaccination of young children. Pediatrics. 2003;111(5 pt 2):1215-1218

6)World Health Organization: Washington DC: Centre for Global Development; Immunization, vaccines and biologicals: Centre for Global Development: Making Markets for vaccines: from ideas to actions2006.

7)Emadi NA, Falamarzi S, AlKuwari MG, Al-Ansari A. Patients' Satisfaction with Primary Health Care Services in Qatar. Middle East J Fam Med. 2009;7:9.

8)Bahrampour and Zolala (2005): Patient satisfaction and related factors in Kerman hospitals. Eastern Mediterranean Health J; 11:905-12.

9)Gadallah M, Zaki B, Rady M, Anwer W, Sallam I: Patient satisfaction with primary health care services in two districts in Lower and Upper Egypt. East Mediterr Health J2003; 9:422-430

\section{0) Abbas A: Maternal} satisfaction about childhood immunization in primary

No. 3

July 
health care center, Egypt. Pan Afr Med J. 2014; 18: 157.

11) Udonwa NE1, Gyuse AN1, Etokidem AJ2, Ogaji DST2: Client views, perception and satisfaction with immunisation services at Primary Health Care Facilities in Calabar, South-South Nigeria .Asian Pacific Journal of Tropical Medicine.2010; 298-301.

12) Sabry A,Salem. M. D: Patient Satisfaction With Primary Health Care Services in Qassim Province ,Saudia Arabia. the Egyptian journal of community.2010; Vol 28 No 3

13) Nath B, Jai V. Singh, Shally Awasthi1, Vidya Bhushan, Shivendra K. Singh and Vishwajeet Kumar : Client Satisfaction with Immunization Services in Urban Slums of Lucknow District. Indian Journal of Pediatrics, Volume 76-May, 2009.

14) Ossai EN:Client satisfaction in immunization services in Urban and Rural primary health care centers of a south eastern states in Nigeria. Niger J Pead.2014; 41(4):375-382.
15) Mithun M Sanghavi :Assessment of routine immunization program at primary health center in Jamnagar. National Journal of Medical Research Volume 3 | Issue 4 | Oct Dec 2013.

16)Dewedar S: Thesis in evaluation of knowledge age, attitude and practice of primary H.C physicians concerning the PHC concepts with a special emphasis on child JMM as one of the main elements. Suez Canal university,1995.

17)Performance Needs Assessment of Basic Health Care Workers in immunization.

http://www.searo.who.int/india/top ics/routine_immunization/Report_ on_Performance_Needs_Assessme nt_of_HWs_2005.pdf

18) Barrera L, Pierson $S$,Trumbo, Bravo $P$ and Martha A: From the parents' perspective: a usersatisfaction survey of immunization services in Guatemala . BMC Public Health, 2014; $14: 231$ http://www.biomedcentral.com/14 $71-2458 / 14 / 231$ 
Table (1): The mean scores for satisfaction and its different components in the 4 PHCs of the study

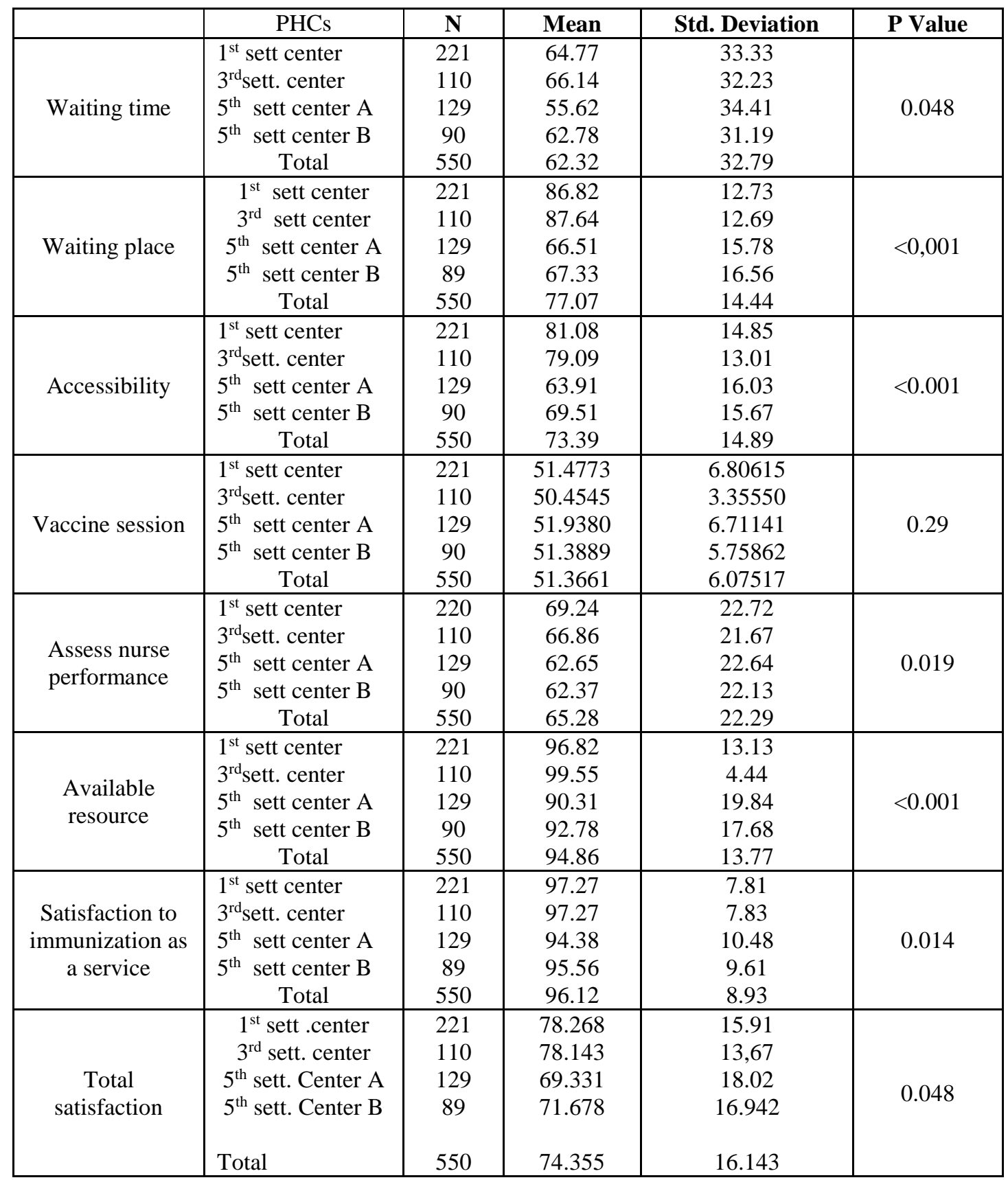

ANOVA test. 
Table (2): Comparison between the 4 PHCs as regard results of checklist evaluating the skills and procedures don by PHC providers

\begin{tabular}{|c|c|c|c|c|c|}
\hline PHC & $\begin{array}{c}\text { Communication } \\
\text { skills and provision } \\
\text { of vaccination } \\
\text { knowledge to } \\
\text { attendant }\end{array}$ & $\begin{array}{c}\text { medical } \\
\text { protocols } \\
\text { inside } \\
\text { vaccination } \\
\text { room }\end{array}$ & $\begin{array}{c}\text { vaccine } \\
\text { handling }\end{array}$ & $\begin{array}{l}\text { administering } \\
\text { immunization }\end{array}$ & $\begin{array}{c}\text { records } \\
\text { procedures } \\
\text { inside } \\
\text { vaccination } \\
\text { room }\end{array}$ \\
\hline e $^{\text {st }}$ settlement & 91.67 & 75.00 & 100.00 & 83.33 & 100.00 \\
\hline $3^{\text {rd }}$ settlement & 50.00 & 75.00 & 100.00 & 66.67 & 75.00 \\
\hline $\begin{array}{c}5^{\text {th }} \text { settlement } \\
\text { center A }\end{array}$ & 50.00 & 62.50 & 100.00 & 66.67 & 75.00 \\
\hline $\begin{array}{c}5^{\text {th }} \text { settlement } \\
\text { center B }\end{array}$ & 50.00 & 50.00 & 90.00 & 66.67 & 75.00 \\
\hline $\begin{array}{c}\text { chi-square } \\
\text { P value }\end{array}$ & $\begin{array}{c}52.64 \\
\mathrm{p}=0.00\end{array}$ & $\begin{array}{c}19,16 \\
p=0.00\end{array}$ & $\begin{array}{c}30.77 \\
p=0.00\end{array}$ & $\begin{array}{c}10.37 \\
p=0.02\end{array}$ & $\begin{array}{c}30.7 \\
\mathrm{p}=0.00\end{array}$ \\
\hline
\end{tabular}

Table (3): Evaluation of vaccine preservation and performance of health workers points not present in the previous checklist.

\begin{tabular}{|c|c|c||}
\hline PHC & $\begin{array}{c}\text { Vaccine } \\
\text { preservation } \\
(\boldsymbol{\%})\end{array}$ & $\begin{array}{c}\text { Performance of health } \\
\text { workers } \\
(\boldsymbol{\%})\end{array}$ \\
\hline \hline $1^{\text {st }}$ settlement & 100 & 85.71 \\
\hline $3^{\text {rd }}$ settlement & 100 & 85.71 \\
\hline $5^{\text {th }}$ settlement center A & 100 & 42.86 \\
\hline $5^{\text {th }}$ settlement center B & 100 & 42.86 \\
\hline chi-square & & 79.8 \\
P value & & $\mathrm{P}=0.00$ \\
\hline
\end{tabular}


Graf(1):Comparison between educated and non educated relative of the child as regards the satisfaction

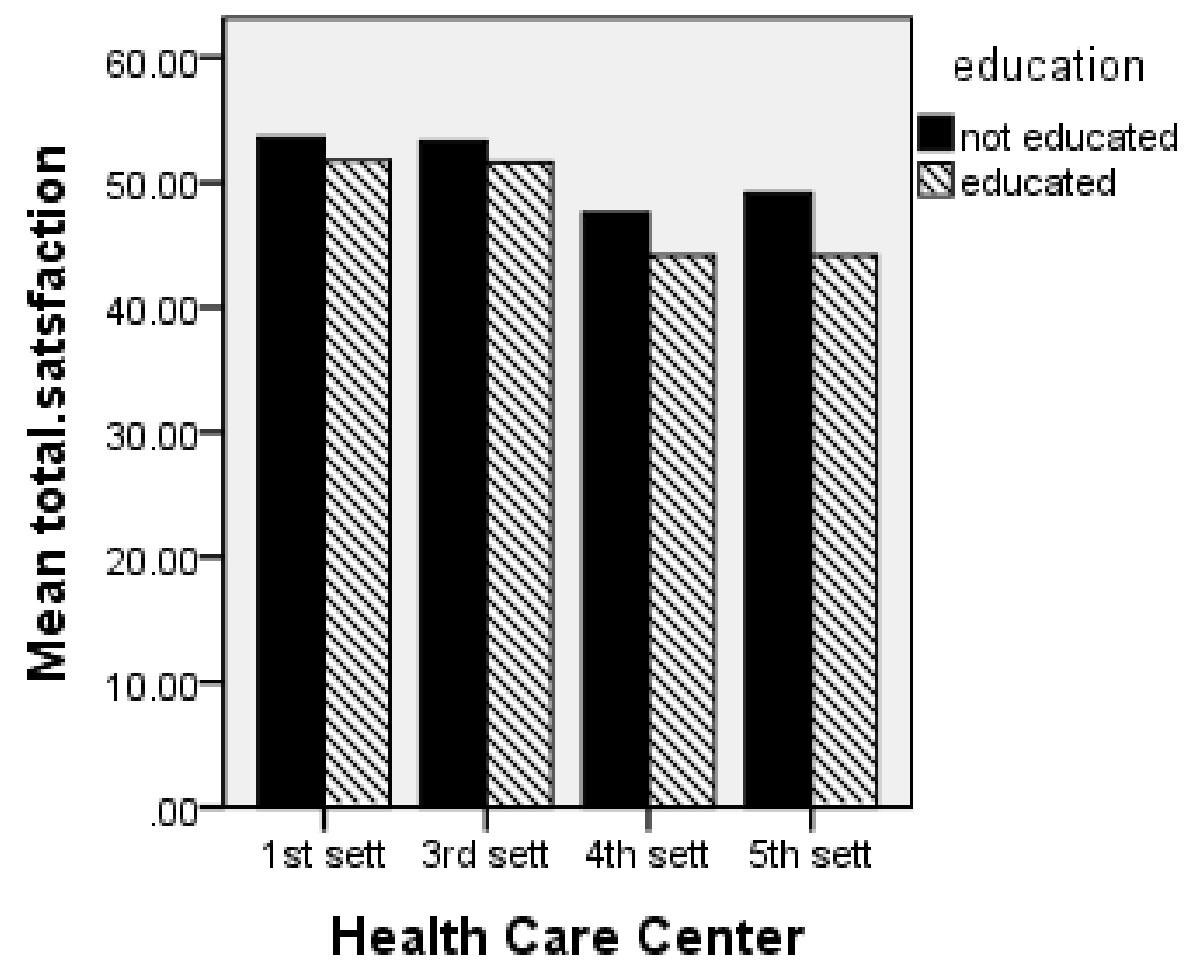

